\title{
Application Challenges and Prospects of Urban Integrated Energy System Planning
}

\author{
Guoxin $\mathrm{He}^{1,2,{ }^{*}}$, Zhihong Yang ${ }^{1}$, Jinda Zhu ${ }^{1}$, Qi Wang ${ }^{2,3}$, Dongmei Yang ${ }^{1}$, Yonghua Chen ${ }^{1}$ and Wei Du ${ }^{1}$ \\ ${ }^{1}$ NARI Group Corporation, NARI Research Institute, 211106 Nanjing, China \\ ${ }^{2}$ Nanjing Normal University, School of Electrical and Automation Engineering, 210046 Nanjing, China \\ ${ }^{3}$ Nanjing Normal University, Jiangsu Gas-Electricity-Interconnected Engineering Laboratory, 210046 Nanjing, China
}

\begin{abstract}
The integrated energy system is the main physical carrier of the energy Internet. Planning is the leading link of its application, which directly affects the necessity and economy of the construction of the project. Firstly, the basic process of integrated energy planning is summarized. Then, three application challenges faced by urban integrated energy system planning are summarized from the perspective of engineering application: integrated energy system refinement modelling, multi-energy load integrated prediction, and expansion of planning boundaries. The corresponding strategies are given for the above challenges, and the development of integrated energy system planning and application is prospected, which provides reference for the staff engaged in urban integrated energy system planning and related investors.
\end{abstract}

\section{Introduction}

As China is now in the critical period of promoting newtype urbanization, its energy resources and ecological environment are faced with unprecedented pressure. Besides, the energy using mode featuring high input, consumption and emission is in urgent need of an upgrading into an intensive, low-carbon and clean mode [1]. As an effective method of promoting energy transformation and upgrading, the "internet plus" intelligent energy (Energy Internet) aims to promote energy system delayering through internet technology, reform energy production and consumption mode, improve energy using efficiency and realize energy conservation and emission reduction, [2-6]. But in terms of the application status, the Energy Internet still has long way to go to achieve scale-based promotion and commercialized application. Main problems include difficult transboundary coordination, unclear profiting mode, lack of key technologies, etc.

The integrated energy system is the main physical carrier of the energy Internet. Planning is the leading link of its application, which directly affects the necessity and economy of the construction of the project. As the integrated energy system includes different energy forms like electricity, cool air, heat, gas and so on, the optimal economic benefits can be obtained at the lowest cost of restoration and construction through conducting objective and integral evaluation for user diversity based on reasonable planning, carrying out sufficient investigation for users' present energy and resource endowment, natural environment conditions, existing infrastructure and the market environment, and proposing the best plan on the basis of users' actual situation.

Existing studies are mostly theoretical analysis while further sorting out is needed for the practical challenges and application effect of integrated energy system planning. In terms of research overview, literature [7] summarizes the key technical problems including multienergy coupling theory, load forecasting method, optimization modelling and solution in regional integrated energy system planning. Besides, the problems and technical difficulties are analyzed from the perspective of theoretical research. As for the planning methods, literature [8] faces new-type production zones and establishes mixed-integer linear programming models with minimum annual cost as the objective function. But linear modelling is mainly based on equipment unit without considering network constraint model. Though the solution difficulty is reduced, it lacks instructive effect on practical projects.

Three application challenges faced by urban integrated energy system planning are summarized from the perspective of engineering application: integrated energy system refinement modelling, multi-energy load integrated prediction, and expansion of planning boundaries. The corresponding strategies are given for the above challenges, and the development of integrated energy system planning and application is prospected, which provides reference for the staff engaged in urban integrated energy system planning and related investors.

\section{Planning process}

\subsection{Urban integrated energy system architecture}

\footnotetext{
* Corresponding author:
} 


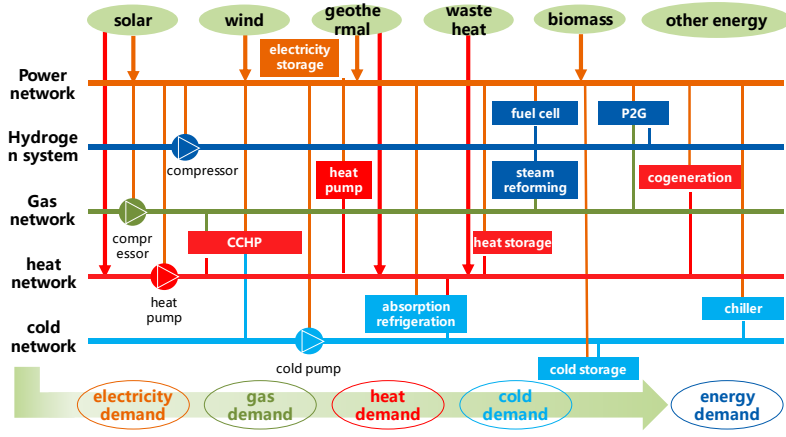

Fig. 1. Urban integrated energy system (IES) architecture.

As is shown in Figure 1, the urban integrated energy system mainly consists of supply network (electricity supply, gas supply (natural gas), heat/cool air supply, hydrogen energy system, etc.), the energy conversion link (joint supply of cool air, heat and electricity, electric boiler, heat pump, absorption refrigeration, etc.), the energy storage link (electricity storage, heat storage, cool energy storage, etc.) and terminal energy using unit. With a comprehensive use of advanced information and physics integration technology and management mode in the urban area, it integrates multiple clean energies including solar energy, wind energy, geothermal energy, biomass energy, etc., and realizes integrative planning, operation optimization and flexible transaction among different energy subsystems, which not only satisfies users' diverse demands but also improves the comprehensive efficiency of energy using.

\subsection{Basic process of IES planning}

The basic objective of integrated energy system planning is to seek the lowest cost combination of energies and resources including electricity, cool air, heat and gas while guaranteeing safety, reliability and high efficiency, thereby meeting users' energy-using demand and satisfying the energy conservation requirements in specific areas in the regulated period (generally 5 to 20 years). As is shown in Figure 2, the basic planning process includes the following steps:

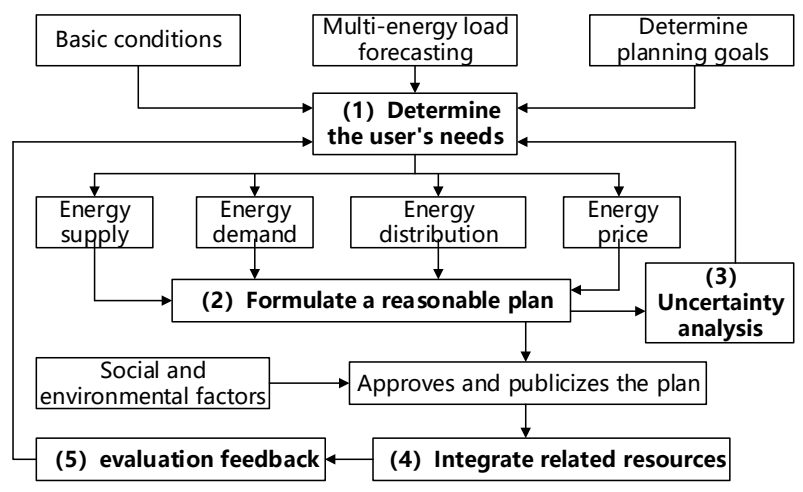

Fig. 2. Basic process of IES planning.

(1) Users' comprehensive energy demands: planners should investigate and study the basic conditions of users' energy facilities. Users with strong comprehensive energy demand usually have problems such as single energy form, extensive energy using mode, lack of energy and resource sufficiency. An overall analysis for present basic conditions can make the target clearer. Traditional urban energy planning separates electricity planning, heat supply planning and gas planning, which leads to the mutual independence of the load prediction of different energy subsystems. By contrast, the multienergy load forecasting takes urban electrical load, heat load and gas load into integrate consideration, which is good for exploiting the coordinative and complementary potentials among electricity, cool air, heat and gas. Based on what is talked above, specific objectives for planning are made, mainly including safety and reliability level and economic and environmental benefit prediction. In addition, the results of step 3 and step 5 should be taken into consideration.

(2) Reasonable and integrated energy system planning scheme: after making clear users' basic energy demands, energy supply mode should be further determined, namely, the layout, scale and quantity of power source, gas source, cool air source and heat source. Then, decide function divisions and calculate the supply scope of each energy; in the aspect of transmission and distribution, the level, arrangement and parameter of the power grid, gas grid, heat grid and ancillary facilities should all be taken into consideration and the selection, capacity and parameter of comprehensive energy network coupling conversion device (joint supply of cool air, heat and electricity) ought to be made clear; take into consideration the supply cost and energy using price and use it as the basic elements of planning scheme economy calculation.

(3) Uncertainty analysis: planners should analyze the potential uncertain factors having influence on the feasibility of planning schemes. For example, the change of terminal energy using price may lead to expected gap of the planning's economic interests; the management power of electricity grid, gas grid and heat grid which is separate may increase the difficulty of management. The results of uncertainty analysis are an important reference for users to assess and recognize the planning scheme. It influences the demand analysis in Step (1).

(4) The results of uncertainty analysis are an important reference for users to assess and recognize the planning scheme. It influences the demand analysis in Step 1. Integrate relevant resources in accordance with the planning scheme: elements such as society and environment must be taken into consideration in comprehensive energy planning; the schemes, after being approved and publicized by the competent departments, are used as the basis of labor force, finance and material integration and project construction.

(5) Project construction, operation and evaluation feedback: though planning is conducted in early phase, its influence goes through the whole process of project construction, operation and evaluation. The evaluation of operation situation indirectly reflects the planning effect 
and project rationality and influences users' investment decision.

\section{Application challenges for planning}

As is shown in Figure 3, the challenges that urban integrated energy system planning is faced with mainly include three aspects: (1) the planning model is coarsegrained while lacking delicacy. As the integrated energy system covers different energy forms including electricity, cool air, heat and gas, current research only conducts simplification at the system modelling level in order to reduce the difficulty of planning solution. Besides, as the uncertainty analysis which influences model accuracy is absent, its credibility isn't strong enough. (2) No integration is conducted for electricity, cool air, heat and gas prediction and the overall prediction accuracy is relatively low, which can hardly support planners to make effective decision schemes; (3) the planning boundary is still narrow and can't adapt to the user-oriented development trend or satisfy the diverse interest appeals of different parties.

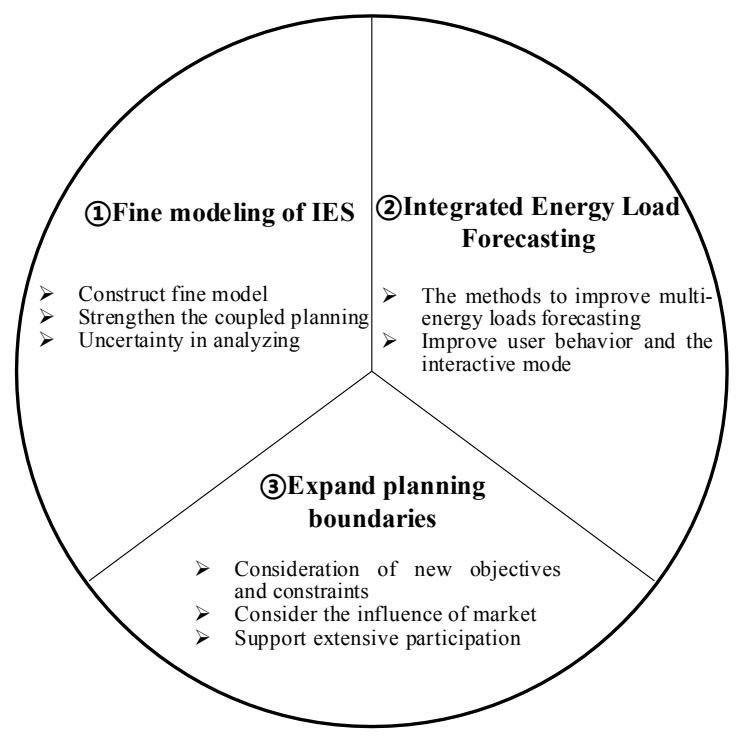

Fig.3. Challenges and strategies for IES planning.

\subsection{IES refinement modelling}

(1) Planning fine-grained model in multiple temporalspatial scales should be constructed

The fine-grained model is originally a term of the computer industry which indicates to subdivide the objects of business models to get more scientific and reasonable object models, [9-11]. As for the comprehensive energy business model, the object models include:

1) The time scale model: on the one hand, the differences of action response time of the electricity system, gas supply system and heat/cool air supply system should be considered. Generally, the response of electricity system is second-based, the fastest; the heat/cool air supply system is minute-based, the second fastest; the gas supply is the slowest. On the other hand, the output models of renewable resources such as photovoltage, wind power, geothermal energy and natural gas in different time scales of year/season/month/day/hour should be established to exploit its coordination and complementarity in multi-time scales. A reasonable method of energy supply ought to be found through comprehensively considering different factors such as storage conditions, system safety, etc.

2) The spatial scale model: the positions and sites of infrastructures such as power source, gas source, cool air source, heat source, electricity grid, gas grid and heat grid are determined by urban planning. In areas with stable electricity, gas, cool air and heat load such as industrial parks, coupled system configuration like cool, heat and electricity combination supply and combined heat and power should be considered according to load distribution and function division; set up regional layered and divisional spatial scale models to determine the coverage of energy supply, distribution of energy network and the transmission capacity of all nodes, and realize spatial coordination and sharing among different energy subsystems.

3) The operation reliability model: traditional planning involves few or no consideration of system operation reliability, mainly because the separate energy subsystems have relatively mature reliability evaluation plans and technical levels. For example, the thermal generator set, after long-term development, is sure to meet the demands for energy while ensuring operation reliability. The new energy production technologies such as solar energy, wind energy and geothermal energy have characteristics of intermittency and volatility while the new energy conversion and transmission technology such as flexible ac/dc power transmission system and gas-based cool air, heat and electricity joint supply system haven't been used extensively. The problem of system reliability brought about after the new-type equipment is extensively interconnected should be fully considered in the planning stage, and whether the selected energy and resource combination can meet the demand for long-term stable operation should be assessed.

(2) The coupled planning of different energy subsystems should be strengthened

The application value of the integrated energy system can be demonstrated only by ensuring reliability, optimizing the entire link of energy output-transmissiondistribution-use and reducing cost to the maximum extent. The key point lies in strengthening the coupled planning among the energy subsystems of electricity, cool air, gas and heat, which specifically includes:

1) Strengthen information sharing and exchanging: though some existing rules of the energy subsystems planning have been formed, some essential data and 
information exchanges are needed to promote the integrated energy system to be more overall and accurate. For example, the best installation positions and scales of electricity storage, heat storage, electric boiler and combined heat and power can be easily determined in electricity grid and heat grid through data sharing; timely sharing of the state information of power and heat storing can obviously improve the energy management level of the entire integrated energy system.

2) Evaluate system sufficiency: integrated energy system reliability analysis includes safety analysis and sufficiency analysis. In the planning stage, focuses are given to the offline system static safety analysis, which has relatively much accumulation. So, no more detailed description is offered here. Sufficiency demonstrates the system's ability of meeting users' demands, such as the ability of maintaining transient and medium-and longterm energy supply and demand balance after being interfered. Besides, it represents the economic characteristics of system technology. If the sufficiency is too large, the system has strong reliability while high cost, which causes waste; if the sufficiency is small, the system costs less but isn't reliable and cannot meet users' demand for energy. Coupled planning should take the system sufficiency into consideration, or the economic efficiency of the technology used in the plan cannot be measured.

\section{(3) The uncertainty in analyzing and processing planning}

As is shown in Table 1, much uncertainty exists in the urban integrated energy system planning, whose potential influences are addressed by means of scenario analysis and stochastic analysis. In practical application, extreme situations are assumed such as policy cancellation, energy price increase, storage price decrease, to establish risk index system and compare the risk degrees of different planning in combination with the weighting method. Compared with the single energy system, the comprehensive one faces more complicated uncertain factors, such as the growth of distributed energy system scale, volatility superposition of multienergy loads including electricity, gas, cool air and heat. On the one hand, new uncertain influence factors should be exploited and the risk index system ought to be updated. On the other hand, new processing methods like combined analysis can be used to solve the uncertainty problem in the process of planning.

Table 1. Uncertain factors in IES planning.

\begin{tabular}{|c|c|c|}
\hline Uncertainty factors & $\begin{array}{c}\text { Current } \\
\text { method }\end{array}$ & $\begin{array}{c}\text { Correspondin } \\
\text { g link }\end{array}$ \\
\hline Policy change & $\begin{array}{c}\text { Scene } \\
\text { analysis }\end{array}$ & $\begin{array}{c}\text { Energy } \\
\text { transmission }\end{array}$ \\
\hline $\begin{array}{c}\text { Supply and demand } \\
\text { changes on both sides }\end{array}$ & $\begin{array}{c}\text { Scene } \\
\text { analysis }\end{array}$ & $\begin{array}{c}\text { Energy } \\
\text { transmission }\end{array}$ \\
\hline $\begin{array}{c}\text { Long-term economic } \\
\text { trends }\end{array}$ & $\begin{array}{c}\text { Scene } \\
\text { analysis }\end{array}$ & $\begin{array}{c}\text { Energy } \\
\text { adequacy }\end{array}$ \\
\hline
\end{tabular}

\begin{tabular}{|c|c|c|}
\hline $\begin{array}{c}\text { Population movements } \\
\text { and increases }\end{array}$ & $\begin{array}{c}\text { Scene } \\
\text { analysis }\end{array}$ & $\begin{array}{c}\text { Energy } \\
\text { transmission }\end{array}$ \\
\hline $\begin{array}{c}\text { Energy price } \\
\text { fluctuations }\end{array}$ & $\begin{array}{c}\text { Scene } \\
\text { analysis }\end{array}$ & $\begin{array}{c}\text { Energy } \\
\text { adequacy }\end{array}$ \\
\hline $\begin{array}{c}\text { Technological progress } \\
\text { (e.g. energy storage) }\end{array}$ & $\begin{array}{c}\text { Scene } \\
\text { analysis }\end{array}$ & $\begin{array}{c}\text { Energy } \\
\text { transmission }\end{array}$ \\
\hline weather change & $\begin{array}{c}\text { Stochastic } \\
\text { analysis }\end{array}$ & $\begin{array}{c}\text { Energy } \\
\text { transmission }\end{array}$ \\
\hline Large unit failure & $\begin{array}{c}\text { Stochastic } \\
\text { analysis }\end{array}$ & $\begin{array}{c}\text { Energy } \\
\text { transmission }\end{array}$ \\
\hline
\end{tabular}

\subsection{Integrated Energy Load Forecasting}

(1) The methods to improve multi-energy loads including electricity, gas, cool air and heat

Different from the power system, the urban integrated energy system load prediction has two features: (1) in addition to electricity demand quantity, the loads also include demands of gas, cool air and gas sources; (2) besides following the traditional prediction methods of power load, heat load and gas load, other factors such as the long-term development of power source, heat source and gas source, energy policies, environmental policies, urban development planning and social economic development situations should all be considered. In combination with the load density method, index method, etc., the multi-energy total load $\mathrm{Q}_{\text {total }}$ can be classified into three categories based on user types, including industrial load $\mathrm{Q}_{\mathrm{i}}$, living load $\mathrm{Q}_{1}$ and building load $\mathrm{Q}_{\mathrm{b}}$. Calculation is based on formula (1):

$$
Q \text { total }=Q i+Q 1+Q b
$$

The loads consist of power load, gas load, cool air load and heat load. Let's take the industrial load as an example:

$$
\begin{gathered}
\mathrm{Qi}=[Q \mathrm{e}, Q \mathrm{~g}, Q \mathrm{~h}] \\
Q \mathrm{e}=S \times D \\
Q \mathrm{~g}=10^{3} G_{y} H_{1}^{y} \eta^{\prime} / H_{1} \eta \\
Q \mathrm{~h}=\sum_{i=1}^{n} q \times A \times 10^{-3}
\end{gathered}
$$

In the formula, $\mathrm{Q}_{\mathrm{e}}, \mathrm{Q}_{\mathrm{g}}$ and $\mathrm{Q}_{\mathrm{h}}$ respectively indicate electricity consumption, gas consumption and heat consumption; $\mathrm{S}$ indicates the population in industrial zones (or building area and land area); D is electricity consumption per capita (the electricity density of building area or land area); $\mathrm{G}_{\mathrm{y}}$ is the annual gas consumption of other fuels; $\mathrm{H}_{1}{ }_{1}$ is the low heating value of other fuels; $\mathrm{H}_{1}$ is the low heating value of gas; $\eta$ ' is thermal efficiency of equipment of other fuels; $\eta$ is the thermal efficiency of gas burning device; $q$ is the industrial heat load index; $\mathrm{A}$ is the industrial land areas of different types; $i$ is industrial types.

The combined certainty load prediction method is often used in project practice. But in fact, the development and change rules of the integrated energy system load are quite complicated and often influenced by many factors. Therefore, the uncertainty prediction methods based on analogy and correspondence should be 
introduced, such as the fuzzy neural network prediction method and artificial intelligence prediction method based on in-depth learning. Through such methods, more credible load prediction results can be obtained and more elaborate energy use portraits can be established. No more detailed description of this is offered in this thesis.

\section{(2) Improve user behavior and the interactive model}

In the era of information, users have access to diversified services which are unprecedented. The influences of user behaviors on integrated energy system keep increasing, which are specifically demonstrated in as follows.

1) Digital influence and users' energy consumption habit change: users' demands for online services promote the digitalization progress of energy systems. For instance, the energy use devices like air-conditioner and electric water heater are becoming more intelligent. They make it easier for users to understand and control their energy consumption. By changing users' energy use habits, digitalization changes the growth track of energy demand as well as the load shapes in different periods.

2) User behaviors have influence on the integrated energy system operation reliability: traditional energy planning seldom considers the interactive model between users and energy network. For example, multiple electric vehicles charge at the same time, which causes overload and problems in power supply reliability; besides, as different users have different personalities, demands and preferences, modelling should be conducted for their behaviors when planning and the potential influence on system reliability should be analyzed.

3) The application of user-end new technologies proposes new challenges for the planning: influenced by technological progress and policy promotion, the permeability of distributed energy systems like distributed photo-voltage and distributed energy storage keeps increasing, which causes huge challenges to the energy service providers to make the optimal planning and operation plans; as the distributed transaction technologies such as block chain are promoting the innovation and reform of user-end energy transaction mode, profound changes of the supply and demand relationship in the integrated energy system are taking place, due to which the planners need to reexamine and launch a tighter integrative planning.

\subsection{Expansion of planning boundaries}

As is shown in Figure 4, the integrated energy system planning isn't simple combination of the power system, heat system and gas system. It should also include:

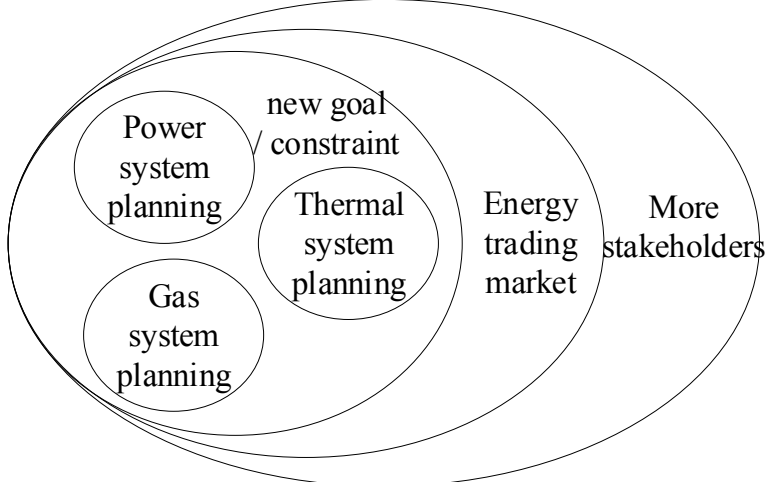

Fig.4. Integrated energy system planning boundary.

(1) Consideration of new objectives and constraints of multi-energy coupled planning

The National New-type Urbanization Plan proposes a new-type urbanization strategy with people-orientation as the core and "urban and rural overall development, urban-rural integration, city-industry interaction, economical and intensive, pleasant living and harmonious development" as basic characteristics. It has become the key development target of urban integrated energy system to improve the comprehensive energy use efficiency and reduce environment pollution. The planning link should take into consideration of both the target of being economical, environmental and safe regulated in traditional electricity, heat and gas systems and realizing energy saving and emission cutting while ensuring reliable operation as well as improving comprehensive energy efficiency. The integrated energy system consists of multiple devices and many links. So, in addition to the essential device constraints, other constraints from multi-energy flow network, multienergy mutual supply safety and environment should also be considered in planning.

(2) Consider the influence of energy trade market on the planning link

Urban energy trade can improve economic efficiency and the reliability of integrated energy system operation. Besides, it can enhance environmental interests and promote employment, [12]. But conflicts may appear between them. Taking photo-voltage as an example, electricity market transaction can promote photo-voltage consumption and improve environmental interests. But due to the intermittency and volatility, the reliability of the integrated energy system may suffer negative influences. Planners should fully evaluate the potential influence of transaction on planning before making decisions. In addition, the pricing mode of energies and resources such as photo-voltage and stored energy in trade is very important as it decides whether the investment cost can be recovered. Also, it is an important basis for making medium- and long-term investment plans. 
(3) Support extensive participation of more interested parties

The integrated energy system planning covers multiple areas and involves many parties. On the one hand, the energy system planning departments of electricity, heat and gas should strengthen their coordination, communication and information sharing. Besides, they should grasp the technical details included in the integrated energy system planning from the overall perspective and solve the difficulties and key points of planning; on the other hand, the energy enterprises, academic groups and energy users are encourage to participate in the planning link, which can help expand the research depth and width of the integrated energy system planning and strengthen the influence and supportive force of the planning, [13-15].

\section{Application prospects}

\subsection{IES planning based on operation- transaction-evaluation}

The integrated energy system planning is closely related to the link operation-transaction-evaluation which is the development trend of urban integrated energy system. In the planning process, there is no need to consider the multiple details in operation control, but the basic operation reliability should be emphasized. Transactions influence energy sufficiency and economic efficiency, and reasonable transaction modes can make the planning more intensive. Evaluation is the examination of planning effects and the evaluation results fed back can facilitate the optimization and updating of the planning. Planning, as the guidance basis of energy system construction, also restricts the objects and scope of operation, trade and evaluation.

\subsection{IES planning based on information-physics- society integration}

Traditional energy system planning is mostly limited to physical system while lacking the consideration for information system and social elements. The integrated energy system planning faced by new-type cities and towns highlights the interactive influence of social elements and energy system, [16]. On the one hand, user behavior models should be established to provide different users with optimized energy using plans and customized services and realize refined management for users. On the other hand, since uncertain policies and supervisions have influence on the integrated energy system development and operation, and user behaviors affect the reliability of system operation, the collection and emphasis on non-energy information should be extended. Besides, the integrated energy system which is based on information should be updated into a form based on intelligence.

\subsection{IES planning based on data mining and data driving}

The emerging data analysis technology can facilitate the refined planning. The planning link involves plenty of data which include the basic device parameters of electricity, gas, cool air and heat energy systems, basic data of energy network, basic condition data of the planned areas, user energy use behavior data, energy macro policy data, energy transaction market data, etc. So, advanced data analysis technologies are needed to exploit and construct the relationship among data, thereby supporting credible planning models. Development of technologies such as big data analysis and in-depth learning provide ideas to solve this problem. Through analyzing the data above, planners can use the technique of data mining to get the demand data of energies including electricity, gas, cool air and heat and optimize project plans, thereby laying a solid foundation for achieving coupled planning.

\section{Conclusion}

The urban integrated energy system is a project coupling energy supply network, energy conversion, energy storage and the terminal energy use unit. It has basic characteristics of multi-energy coordination, separate supply and demand and open transaction. Planning, the guiding link of its implementation, is in the stage of startup. Existing planning faces challenges of too simple models, insufficient load prediction accuracy, limited consideration range, etc., which leads to the facts that planning and operation construction are disjointed, and the planning lacks credibility and guidance and can hardly support the promotion and application of the integrated energy system.

Judging from the perspective of engineering application, the difficulties that the current urban integrated energy system faces are sorted out and corresponding strategies are provided. The integrated energy system planning is a large project which involves quite many uncertain factors. It is sure that it will have closer coupling with the link operation-transactionevaluation. The user-center idea demands that user behaviors and social factors should be taken into consideration in planning, and people orientation is the goal and trend of the urban integrated energy system planning. Besides, as the planning process involves mass data concerning energy system devices of electricity, gas, cool air and heat, network, environment, staff, etc., the technology of big data analysis and mining should be used to facilitate the process. Only by facing up to the different difficulties and challenges in the planning process, can we fully exploit the value of the integrated energy system.

\section{Acknowledgement}

Thanks to National Key Research Program of China "Key Technologies and Applications of Energy Internet for New Towns" (2018YFB0905000) for supporting this paper. 


\section{References}

1. ZHEN Yuping, WANG Dan, WAN Can, et al. Key technology and application of energy internet oriented to new-type towns $[\mathrm{J}]$. Automation of Electric Power Systems, 2019(14).

2. Zeng B, Zhang J, Yang X, et al. Integrated planning for transition to low-carbon distribution system with renewable energy generation and demand response[J]. IEEE Transactions on Power Systems, 2013, 29(3): 1153-1165.

3. Lund $\mathrm{H}$, Werner $\mathrm{S}$, Wiltshire $\mathrm{R}$, et al. 4th Generation District Heating (4GDH): Integrating smart thermal grids into future sustainable energy systems[J]. Energy, 2014, 68: 1-11.

4. Amirnekooei K, Ardehali M M, Sadri A. Integrated resource planning for Iran: Development of reference energy system, forecast, and long-term energy-environment plan[J]. Energy, 2012, 46(1): 374-385.

5. Keirstead J, Samsatli N, Shah N, et al. The implications of CHP planning restrictions on the efficiency of urban energy systems[C]//23rd International Conference on Efficiency, Cost, Optimization, Simulation and Environmental Impact of Energy Systems, Lausanne. 2010.

6. Dong Z, Zhao J, Wen F, et al. From smart grid to energy internet: basic concept and research framework[J]. Automation of electric power systems, 2014, 38(15): 1-11.

7. Cheng Haozhong, $\mathrm{Hu}$ Xiao, Wang Li, et al. Review on research of regional integrated energy system planning $[\mathrm{J}]$. Automation of Electric Power Systems, 2019, 43(7): 2-13.

8. Zhu Xu, Yang Jun, Liu Yuan, et al. A planning method for integrated energy system in parks[J]. Renewable Energy Resources, 2018, 36(10):61-67.
9. Quelhas A, Gil E, McCalley J D, et al. A multiperiod generalized network flow model of the US integrated energy system: Part I-Model description[J]. IEEE Transactions on Power Systems, 2007, 22(2): 829-836.

10. Collins S, Deane J P, Poncelet K, et al. Integrating short term variations of the power system into integrated energy system models: A methodological review $[\mathrm{J}]$. Renewable and Sustainable Energy Reviews, 2017, 76: 839-856.

11. Mirakyan A, De Guio R. Modelling and uncertainties in integrated energy planning[J]. Renewable and Sustainable Energy Reviews, 2015, 46: 62-69.

12. Wang D, Jia H, Hou K, et al. Integrated demand response in district electricity-heating network considering double auction retail energy market based on demand-side energy stations[J]. Applied Energy, 2019, 248: 656-678.

13. Mirakyan A, De Guio R. Integrated energy planning in cities and territories: A review of methods and tools[J]. Renewable and Sustainable Energy Reviews, 2013, 22: 289-297.

14. Bakken B H, Holen A T. Energy service systems: Integrated planning case studies[C]//IEEE Power Engineering Society General Meeting, 2004. IEEE, 2004: 2068-2073.

15. Lin Y, Bie Z. Study on the resilience of the integrated energy system[J]. Energy Procedia, 2016, 103: 171-176.

16. Zhao Junhua, Wen Fusuan, Xue Yusheng, et al. Modelling analysis and control research framework of cyber physical power systems[J]. Automation of Electric Power Systems, 2011, 35(16):1-8. 